\title{
Spinal and supraspinal modulation of visceral sensation
}

\author{
E A Mayer
}

\begin{abstract}
Summary
In response to perceived or experienced change, which is considered threatening to the individual, the central nervous system mounts a stereotypic response which decreases the sensitivity to somatic pain, modulates autonomic nervous system outflow, and activates the hypothalamic-pituitary-adrenal axis. Recent experimental evidence obtained in rats suggests that this response can be associated with an increase in visceral sensitivity. This response of the "emotional motor system" may or may not be associated with the conscious experience of feelings of fear or anxiety. Alterations in some of these response systems (antinociception, vigilance) may be responsible for the characteristic visceral hypersensitivity often seen in patients with functional gastrointestinal disorders.
\end{abstract}

\section{Introduction}

Irritable bowel syndrome (IBS) is a chronic disorder of unknown aetiology characterised by exacerbations and remissions, which presents with symptoms of abdominal pain and discomfort, and alterations in bowel habits. Approximately two thirds of patients report abdominal pain as one of their symptoms, and abdominal pain is reported by $40 \%$ of patients when asked for their most bothersome symptom. ${ }^{1}$ The occurrence of certain stressful life events is the most important factor in symptom exacerbation and in the first development of symptoms..$^{2-5}$ A recent prospective study emphasised that life events of a sustained duration, associated with threats to the patient's security, are most commonly associated with symptom exacerbation. ${ }^{2}$ In addition to these symptoms referred to the digestive system, clinical experience and recent reports in the literature have demonstrated a variety of extraintestinal symptoms in these patients, including other pain syndromes such as fibromyalgia $(\mathrm{FM})^{67}$ and interstitial cystitis. ${ }^{8}$ Furthermore, there is a close overlap between IBS and anxiety disorders. ${ }^{9}$ This article reviews evidence for the involvement of supraspinal pain modulation systems in the reported visceral hypersensitivity in IBS patients. For more information on the general topic of visceral hyperalgesia, the reader is referred to extensive earlier reviews. ${ }^{10} 11$

The article focuses on a model of IBS which emphasises alterations in the response of the central nervous system to perceived threat or fear, and discusses recent concepts related to the neuroanatomical basis of conditioned fear in animals. ${ }^{12}$ Analogous to concepts that have evolved to understand the pathophysiology of panic disorder, the majority of non-constipated
IBS patients show evidence of aversive interoceptive conditioning. ${ }^{13}$ While patients with panic disorder show fear responses to cardiovascular visceral afferent information (that is, their heart rate), non-constipated IBS patients show evidence of physiological fear responses to visceral information arising from the colon, such as fullness or urgency. In contrast with panic attacks, the physiological fear responses in IBS patients are often not associated with the conscious feeling of anxiety. However, in a subset of IBS patients, strong feelings of anticipatory anxiety are clearly associated with not being in the right place when the urge to have a bowel movement occurs. One may therefore assume that IBS patients have learned to associate a neutral stimulus (normal sensations of fullness and urgency, or the context in which such sensations might arise, such as food intake, time of day, leaving the house) with fear. The fear response triggered by a discrete stimulus or by contextual stimuli manifests as exaggerated autonomic responses, an increased vigilance towards visceral afferent information arising from the gastrointestinal tract (in particular the sensation of urgency), and inadequate activation of descending pain modulation systems.

Two principal mechanisms are likely to play a role in the modulation of visceral sensation: selective attention and endogenous pain inhibition. The combination of these two mechanisms allows the brain to focus the attention on sensations arising from a particular body region, such as the rectosigmoid, while tuning out sensory input from all other regions. Focused attention may be related to activation of descending pain facilitatory pathways, while activation of descending pain inhibitory pathways may play a role in the suppression of information from non-attended to body regions. This differential modulation thereby increases the signal to noise ratio for a particular body region. Brain regions that are important in selective attention are regions of the anterior executive attentional system, such as the dorsolateral prefrontal cortex and the anterior cingulate cortex. Brain regions that are important in antinociception are regions belonging to the medial pain system, including the medial thalamus, anterior cingulate, amygdala, and periaqueductal grey (PAG). The PAG projects to spinal dorsal horn neurones via the rostral ventral medulla.

Abbreviations used in this paper: IBS, irritable bowel syndrome; PAG, periaqueductal grey; EMS, emotional motor system; FM, fibromyalgia; NMDA, $\mathrm{N}$-methly-D-aspartate. 


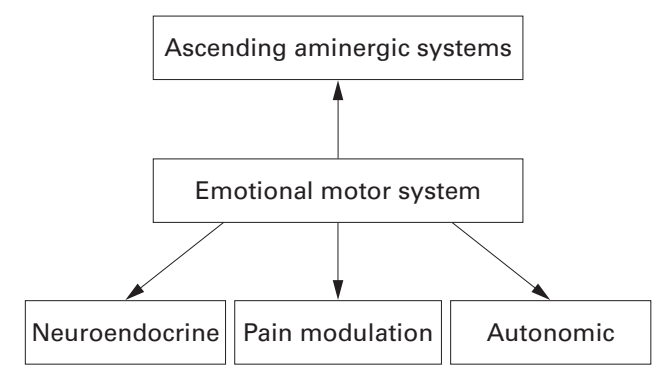

Figure 1 The emotional motor system (EMS).

\section{The emotional motor system}

The close overlap of brain regions concerned with the processing of visceral afferent innervation, arousal, and with central autonomic regulation of gastrointestinal motility and secretory function may have important consequences for the development of IBS symptoms that involve both alterations in perception and autonomic responses to visceral events. The rostral portion of the anterior cingulate cortex represents the visceral motor cortex with projections to a network made up of hypothalamic nuclei, amygdala, and PAG. The output of this network reaches nuclei within the brain stem (rostral ventral medulla, locus coeruleus, raphe nuclei) with important roles in the regulation of pain modulation, arousal, and vigilance. This network has been referred to as the emotional motor system (EMS) $)^{14}$ and relates to a specific set of parallel motor pathways governing somatic, autonomic, antinociceptive, and endocrine responses ${ }^{14}$ to stressors (fig 1 ).

Two components of the EMS play a crucial role in modulation of the pain experience by the organism in the context of conditioned fear: the amygdala and PAG. The amygdala receives information about visceral stimuli not only from the visceral motor cortex; the lateral and basolateral nucleus of the amygdala receive projections from the thalamus, nucleus parabrachialis, and nucleus gigantocellularis. The latter input reaches the amygdala via noradrenergic projections from the locus coeruleus. ${ }^{15}$ The central nucleus of the amygdala sends projections to the lateral and ventrolateral portion of the PAG, mediating opioid and non-opioid mediated analgesia. ${ }^{16-18}$ The connections between the lateral and basolateral nucleus of the amygdala and the central nucleus contain glutaminergic synapses involving both $N$-methly-D-aspartate (NMDA) and non-NMDA receptors. The NMDA receptor is involved in the development of long term potentiation, an important mechanism in the development of emotional memory in the amygdala, in the context of fear conditioning. ${ }^{15}$

\section{Alterations in perception of visceral events}

Enhanced perception of certain visceral stimuli, either as a result of greater sensitivity of visceral afferent pathways or as central amplification of visceral afferent input, has emerged as an important theme in research into panic disorders and into functional gastrointestinal disorders, including IBS. ${ }^{19-21}$ The initial clinical observations that led to the hypothesis of visceral hypersensitivity include the presence of recurring abdominal pain, tenderness during palpation of the sigmoid colon during physical examination, and excessive pain during endoscopic examinations of the sigmoid colon in IBS patients. A variety of studies have reported various aspects of visceral psychophysiology: (1) IBS is associated with hypersensitivity in the upper gastrointestinal tract as well as in the colon $^{22}{ }^{23}$; (2) IBS is associated with a heightened perception of normal intestinal contractions $^{24}$; (3) IBS (unlike FM) is not associated with a generalised hypersensitivity to noxious somatic stimulation ${ }^{25}{ }^{26}$; and (4) the perception of colonic distensions is modifiable by attention, anxiety, and relaxation. ${ }^{27} 28$

Published experimental evidence from studies assessing visceral sensitivity suggest that a variety of perceptual abnormalities in relation to gastrointestinal stimuli may be more frequent in IBS patients. ${ }^{24} 290$ At least two perceptual alterations can be distinguished: (1) hypervigilance towards expected aversive events arising from the viscera; and (2) hyperalgesia which is inducible by sustained noxious visceral stimulation

\section{Hypervigilance}

Hypervigilance is an important component of the integrated response of the nervous system to perceived threat. Important pathways involved in the mediation of arousal and vigilance towards specific sensory stimuli are ascending monoaminergic arousal systems arising from nuclei in the brain stem and forebrain. Emotional reactions are typically associated with intense cortical arousal. Enhanced cortical arousal may be associated with enhanced sensitivity towards visceral sensations associated with a particular emotional experience. Urinary urgency, palpitations, and "butterflies" in the stomach are commonly reported by healthy individuals when experiencing fear. In the following, we will review a series of experimental studies which address the presence of hypervigilance towards visceral stimuli in patients with functional gastrointestinal disorders.

Mertz and colleagues ${ }^{30}$ found that IBS patients had a significantly lower median discomfort threshold for a 30 second rectal balloon stimulus compared with a normal population. If lowered threshold and two other perceptual abnormalities were considered (an abnormal area of sensory referral and/or increased intensity of rectal sensations during balloon distension), $95 \%$ of IBS patients had at least one abnormality. Only $7 \%$ of a control population had at least one of these three sensory findings. Other studies have also found significant perceptual alterations in IBS populations, including lowered discomfort thresholds for balloon distension of the small intestine, colon, and rectosigmoid. 222425293132 Similar findings of hypersensitivity have also been reported for patients with functional dyspepsia ${ }^{20}{ }^{33-36}$ and non-cardiac chest pain. ${ }^{37} 38$ These findings are paralleled by similar findings of target system hypersensitivity in other disorders such as FM and myofascial pain dis- 
order. The majority of reported studies used study designs favouring the assessment of vigilance rather than hyperalgesia or allodynia. The role of cognitive factors, such as selective attention and vigilance, in modulating the perception of visceral input to the brain is also supported by preliminary results from brain imaging studies. ${ }^{39} 40$

\section{Altered endogenous pain modulation of noxious visceral stimuli}

A series of studies have demonstrated that patients with functional gastrointestinal disorders, including those with IBS, show enhanced perceptual responses to repeated noxious distension of the viscera. Analogous findings of inducible visceral hyperalgesia have been demonstrated in the duodenum ${ }^{41}$ and sigmoid colon. ${ }^{42}$ One way to explain these findings is by postulating alteration in the balance of endogenous pain modulation systems, activated by the noxious repetitive stimulus, which in turn modulate spinal cord excitability. ${ }^{1011}$ Brain stem nuclei (rostral ventral medulla) send descending projections to the spinal cord dorsal horn which can facilitate or inhibit the excitability of these neurones receiving afferent input from the viscera. ${ }^{43}$ The balance between these inhibitory and facilitatory influences determines the amount of visceral afferent information that reaches supraspinal centres.

To study the mechanisms underlying the different perceptual responses to the rectosigmoid distension paradigm, we performed functional brain imaging studies in healthy control subjects and IBS patients during the rectosigmoid distension paradigm, using $\mathrm{H}_{2}{ }^{15} \mathrm{O}$ positron emission tomography. Following a train of repetitive sigmoid distensions, control subjects showed greater activation of the PAG and thalamic regions than IBS patients. This differential activation was seen both during rectal distension of moderate intensity $(45 \mathrm{~mm} \mathrm{Hg})$ and during the expectation of such a stimulus, without actual delivery. The percentage of patients reporting pain during the rectal stimulus was significantly greater than that of control subjects. These findings are consistent with the concept that IBS patients show inadequate activation of brain regions concerned with antinociception. This "defect" is seen both during an actual stimulus and during the expectation of such an event. ${ }^{44}$

\section{Alterations in somatic pain perception}

A series of epidemiological studies have confirmed the clinical impression that IBS and other functional pain disorders, such as FM, typically overlap in the same patient, suggesting a common pathophysiology. FM syndrome occurs in $32-70 \%$ of patients with IBS $^{67456}$ and up to $65 \%$ of IBS patients suffer from FM symptoms. ${ }^{6}$ It has been suggested that patients suffering from IBS or FM may share alterations in CNS mechanisms concerned with antinociceptive responses to sensory stimulation. In addition, attentional mechanisms in the brain may determine whether predominant symptom expression involves the musculoskeletal, gastrointestinal system, or both. By comparing somatic and visceral pain perception in patients with IBS, FM, and IBS+FM, Chang et al recently reported the following findings: while IBS patients were hypervigilant to mechanical somatic stimuli, somatic perception alterations were dependent on whether FM was a comorbid condition. ${ }^{47}$ Patients with IBS+FM demonstrated hypersensitivity to somatic stimuli (as described for FM patients without IBS) while patients with IBS alone showed a blunted response. In addition, IBS patients had significantly lower rectal discomfort thresholds compared with IBS+FM patients and healthy controls before and after a noxious sigmoid stimulus. While IBS+FM patients had similar baseline rectal discomfort thresholds to those of controls, they developed rectal hypersensitivity following sigmoid conditioning similar to the phenomenon seen in a subset of IBS patients. In addition, IBS and IBS+FM patients rated the rectosigmoid distension in more unpleasant terms than healthy subjects. These results suggest that IBS patients show visceral hyperalgesia, patients with FM show somatic hyperalgesia, and that the overlap group of IBS+FM show features of both.

\section{Summary and conclusions}

Hypervigilance and alteration in descending pain modulation systems appear to be important components in the development of enhanced visceral sensitivity in IBS patients. In addition, secondary peripheral changes induced by autonomic dysregulation of specific target cells within the gut (such as enterochromaffin cells, enteric neurones, immune cells, and interstitial cells of Cajal) may contribute to the observed visceral allodynia.

Many clinical and pathophysiological observations in IBS patients are consistent with an exaggerated response of the EMS to perceived threat. The close association between sustained threatening life events and symptom exacerbation, the often dramatic analgesic response to anxiolytics and sedatives, the reported autonomic and neuroendocrine responses, and the significant overlap of anxiety disorders with functional gastrointestinal disorders indicate a prominent role of supraspinal mechanisms in the modulation of visceral sensitivity and pain. An important component of the EMS is opioid and non-opioid dependent pain modulation pathways which are triggered by inputs from the amygdala to specific columns within the PAG. The preliminary results from brain imaging studies demonstrating decreased activation of these two structures during rectal distension and during anticipation of such distension strongly implicate failure of activation of such pain modulation systems in IBS patients. In addition, preliminary results from studies assessing hypervigilance with electroencephalographic techniques ${ }^{40}$ have provided convincing evidence for the presence of hypervigilance towards certain visceral afferent input.

\footnotetext{
1 Lembo T, Naliboff B, Munakata J, et al. Symptoms and visceral perception in patients with pain-predominant irritable bowel syndrome. Am 7 Gastroenterol 1999;94:1320-6.

2 Bennett EJ, Tennant CC, Piesse C, et al. Level of chronic life stress predicts clinical outcome in irritable bowel syndrome. Gut 1998;43:256-61.
} 
3 Gwee KA. The role of psychological and biological factors in postinfective gut dysfunction. Gut 1999;44:400-6.

Whitehead WE, Crowell MD, Robinson JC, et al. Effects of stressful life events on bowel symptoms: subjects with irritable bowel syndrome compared with subjects without bowel dysfunction. Gut 1992;33:825-30.

5 Whitehead WE, Bosmajian L, Zonderman AB, et al. Symptoms of psychological distress associated with irritable bowel syndrome. Gastroenterology 1988;95:709-14

6 Veale D, Kavanagh G, Fielding JF, et al. Primary fibromyalgia and the irritable bowel syndrome: different expressions
of a common pathogenetic process. $B r f$ Rheumatol 1991;30:220-2.

7 Triadafilopoulos G, Simms RW, Goldenberg DL. Bowel dysfunction in fibromyalgia syndrome. Dig Dis Sci 1991;36: 59-64.

8 Alagiri M, Chottiner S, Ratner V, et al. Interstitial cystitis: unexplained associations with other chronic disease and pain syndromes. Urology 1997;49:52-7.

9 Lydiard RB, Falsetti SA. Experience with anxiety and depression treatment studies: Implication for designing IBS clinical trials. Am f Medicine 1999;107(Suppl 1):65-73.

10 Mayer EA, Gebhart GF. Basic and clinical aspects of visceral hyperalgesia. Gastroenterology 1994; 107:271-93.

11 Mayer EA, Gebhart GF, Mayer EA, et al, eds. Basic and clinical aspects of chronic abdominal pain. New York: Elsevier, 1993:3-28

12 Fendt M, Fanselow MS. The neuroanatomical and neurochemical basis of conditioned fear. Neurosci Biobehav Rev 1999;23:743-60.

13 Razran G. The psychopathology of interoceptive stimulation. Proc Annu Meet Am Psychopathol Assoc 1965;53:6282.

14 Holstege G, Bandler R, Saper CB, et al, eds. The emotional motor system, 107 edn. Amsterdam: Elsevier, 1996:3-6.

15 Fendt M, Koch M, Schnitzler HU. Amygdaloid noradrenaline is involved in the sensitization of the acoustic startle response in rats. Pharmacol Biochem Behav 1994;48:307respons.

16 Manning $\mathrm{BH}$, Mayer DJ. The central nucleus of the amygdala contributes to the production of morphine antinociception in the formalin test. Pain 1995;63:141-52.

17 Manning BH, Mayer DJ. The central nucleus of the amygdala contributes to the production of morphine antinociception in the rat tail-flick test. I Neurosci 1995;15 8199-213.

18 Helmstetter FJ, Tershner ST, Poore LH, et al. Antinociception following opioid stimulation in the basolatera amygdala is expressed through the periaqueductal gray and rostral ventromedial medulla. Brain Res 1998;779:104-18.

19 Mayer EA, Raybould HE. Role of visceral afferent mechanisms in functional bowel disorders. Gastroenterology 1990;99:1688-704.

20 Mertz H, Fullerton S, Naliboff B, et al. Symptoms and visceral perception in severe functional and organic dyspepsia.
Gut 1998;42:814-22.

21 Richter JE, Barish CF, Castell DO. Abnormal sensory perception in patients with esophageal chest pain. Gastroenperception in patients wi

22 Trimble KC, Farouk R, Pryde A, et al. Heightened visceral sensation in functional gastrointestinal disease is not site-specific. Evidence for a generalized sitivity. Dig Dis Sci 1995;40:1607-13.

23 Constantini M, Sturniolo GC, Zaninotto G, et al. Altered esophageal pain threshold in irritable bowel syndrome. Dig Dis Sci 1993;38:206-12

24 Kellow JE, Eckersley CM, Jones MP. Enhanced perception of physiological intestinal motility in the irritable bowe syndrome. Gastroenterology 1991;101:1621-7.

25 Whitehead WE, Holtkotter B, Enck P, et al. Tolerance for rectosigmoid distention in irritable bowel syndrome. Gastroenterology 1990:98:1187-92.
26 Cook IJ, Van Eeden A, Collins SM. Patients with irritable bowel syndrome have greater pain tolerance than normal subjects. Gastroenterology 1987;93:727-33.

27 Accarino A, Azpiroz F, Malagelada JR. Attention and distraction: Effects on gut perception. Gastroenterology 1997;113:415-22.

28 Ford MJ, Camilleri M, Zinsmeister AR, et al. Psychosensory modulation of colonic sensation in the human transverse and sigmoid colon. Gastroenterology 1995;109:1772-80.

29 Bradette M, Delvaux M, Staumont G, et al. Evaluation of colonic sensory thresholds in IBS patients using a barostat. Dig Dis Sci 1994;39:449-57.

30 Mertz H, Naliboff B, Munakata J, et al. Altered rectal perception is a biological marker of patients with irritable bowel syndrome. Gastroenterology 1995;109:40-52.

31 Prior A, Sorial E, Sun W-M, et al. Irritable bowel syndrome: differences between patients who show rectal sensitivity and those who do not. Eur f Gastroenterol Hepatol 1993;5:343-9.

32 Ritchie J. Pain from distension of the pelvic colon by inflating a balloon in the irritable colon syndrome. Gut 1973;14: $125-32$.

33 Mearin F, Cucala M, Azpiroz F, et al. The origin of symptoms on the brain-gut axis in functional dyspepsia. Gastroenterology 1991;101:999-1006.

34 Bradette M, Pare P, Douville P, et al. Visceral perception in health and functional dyspepsia. Crossover study of gastric distension with placebo and domperidone. Dig Dis $\mathrm{Sci}_{\mathrm{i}}$ 1991:36:52-8.

35 Mearin F, Cucala M, Azpiroz F, et al. The origin of symptoms on the brain-gut axis in functional dyspepsia. Gastroenterology 1991;101:999-1006.

36 Bradette M, Pare P, Douville P, et al. Visceral perception in health and functional dyspepsia. Crossover study of gastric distension with placebo and domperidone. Dig Dis $\mathrm{Sci}$ 1991;36:52-8.

37 Cannon RO, Benjamin SB. Chest pain as a consequence of abnormal visceral nociception. Dig Dis Sci 1993;38:193-6.

38 Richter JE, Bradley LA, Mayer EA, et al, eds. Basic and clinical aspects of chronic abdominal pain. Amsterdam: Elsevier, 1993:4.

39 Silverman DH, Munakata JA, Ennes H, et al. Regional cerebral activity in normal and pathological perception of visceral pain. Gastroenterology 1997;112:64-72.

40 Hollerbach S, Tougas G, Frieling T, et al. Cerebral evoked responses to gastrointestinal stimulation in humans. Crit Rev Biomed Eng 1997;25:203-42.

41 Holtmann G, Neufang-Hueber J, Goebell H, et al. Influence of repeated phasic distension on gastric mechanosensory function on subjects (non-patients) with and without functional dyspepsia. Gastroenterology 1998;114:A151.

42 Munakata J, Naliboff B, Harraf F, et al. Repetitive sigmoid stimulation induces rectal hyperalgesia in patients with irritable bowl syndrome. Gastroenterology 1997;112:55-63.

43 Fields HL, Heinricher MM, Mason P. Neurotransmitters in nociceptive modulatory circuits. Anпu Rev Neurosci 1991; 14:219-45.

44 Mayer EA, Liu M, Munakata J, et al. Regional brain activity before and after visceral sensitization. Soc Neurosci 1998;24:529.

45 Sivri A, Cindas A, Dincer F, et al. Bowel dysfunction and irritable bowel syndrome in fibromyalgia patients Clin Rheumatol 1996;15:283-6.

46 Sperber A, Atzmon Y, Weitzman I, et al. The prevalence and implications of irritable bowel syndrome in female fibromyalgia patients. Gastroenterology 1998;114:841.

47 Chang L, Mayer EA, FitzGerald L, et al. Altered perception of somatic stimuli by IBS patients with and without fibromyalgia (FM). Gastroenterology 1997;112:709. 